\title{
Accuracy of self-reported waist and hip measurements in 4492 EPIC-Oxford participants
}

\author{
Elizabeth A Spencer*, Andrew W Roddam and Timothy J Key \\ Cancer Research UK Epidemiology Unit, University of Oxford, Radcliffe Infirmary, Oxford OX2 6HE, UK
}

Submitted 26 August 2003: Accepted 7 January 2004

\begin{abstract}
Objective: To assess the accuracy of self-reported waist and hip circumferences and the waist-to-hip ratio (WHR) by comparison with measured waist and hip circumferences and WHR in a sample of middle-aged men and women.

Design: Analysis of measured and self-reported waist and hip data from participants in the Oxford cohort of the European Prospective Investigation into Cancer and Nutrition (EPIC-Oxford).

Participants: Four thousand four hundred and ninety-two British men and women aged $35-76$ years.

Results: Spearman's rank correlation coefficients between measured and self-reported waist, hip and WHR were $0.80,0.74$ and 0.44 , respectively, for men and $0.83,0.86$ and 0.62 for women. Waist was underestimated, on average, by 3.1 (standard deviation (SD) 5.6 ) $\mathrm{cm}$ in men and 1.9 (SD 5.4) $\mathrm{cm}$ in women. The extent of underestimation was greater in participants with larger waists, older participants and women with greater body mass index (BMI). Hip was underestimated by a mean of 1.8 (SD 4.9) cm in men and 1.2 (SD 4.5) cm in women; the extent of underestimation was greater in participants with larger hip circumference and older participants. On average, WHR was underestimated by less than $2 \%$ by men and women; the extent of underestimation was greater among those with larger WHR, older people and those with greater BMI. Using self-reported values, the proportion of classification to the correct tertile was over $65 \%$ for waist and hip measurements. For WHR this proportion was 50\% among men and 60\% among women.

Conclusions: Self-reported waist and hip measurements in EPIC-Oxford are sufficiently accurate for identifying relationships in epidemiological studies.
\end{abstract}

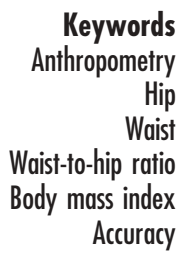

Waist and hip measurements are of interest in epidemiological studies as indicators of the amount and distribution of body fat in individuals, factors of interest in relation to the risk of cancer. In addition, waist circumference alone has been proposed as a means to identify those at increased risk of cardiovascular disease ${ }^{1}$. In large studies, anthropometric data are often collected by self-report. Previous studies generally showed self-reported waist and hip measurements to correlate highly with measured values and average discrepancies between self-reported and measured waist and hip measurements to be $l_{0}{ }^{2-8}$. These studies each included fewer than 400 participants and all but one were performed in the USA. This study compares self-reported and measured waist and hip circumference and waist-to-hip ratio (WHR) data from 4492 participants in the Oxford cohort of the European Prospective Investigation into Cancer and Nutrition (EPIC-Oxford).

\section{Participants and methods}

This study used data from a subset of EPIC-Oxford participants for whom both self-reported and measured waist and hip circumferences were available. Between 1993 and 1999, 5140 middle-aged EPIC-Oxford participants who were recruited via general medical practices in the UK completed a diet and lifestyle questionnaire?. In response to the questions 'What is your present waist size?' and 'What is your present hip size?' participants recorded their waist and hip measurements, in either imperial or metric units. No instructions as to how to take the measures were given to the participants. The questionnaires were optically scanned and the data stored on computer. Waist and hip measurements were rounded to the nearest $\mathrm{cm}$. Within a few weeks of completing the questionnaire, the participants kept an appointment at the general practice and a nurse measured their waist and hip circumferences, following a standard protocol. Waist and 
hip were measured in light indoor clothing, with empty pockets and with tight garments removed. Waist was measured midway between the lower rib margin and the iliac crest. Hip measurement was made at the maximum circumference of the buttocks. Circumferences were recorded to the nearest $\mathrm{cm}$.

Discrepancies between measured and self-reported waist and hip circumferences of over $5 \mathrm{~cm}$ were checked for data-entry errors. Body mass index (BMI) was calculated as measured weight in $\mathrm{kg}$ divided by the square of measured height in $\mathrm{m}$. Data were excluded if BMI was under $15 \mathrm{~kg} \mathrm{~m}^{-2}$ or over $60 \mathrm{~kg} \mathrm{~m}^{-2}$ (one woman excluded). After exclusions owing to recording error, missing or extreme values as defined above, data were available for 1588 men and 2904 women.

Data were analysed separately for men and women. Coefficients for Spearman's rank correlation between measured and self-reported waist, measured and selfreported hip and measured and self-reported WHR were calculated, and Student's paired samples $t$-test was used to compare the means of measured and self-reported values. Analysis of variance was used to examine how the differences (measured minus self-reported values) varied with sex-specific tertile of measured waist and hip and BMI, and with age group at recruitment (35-49, 50-59, 60-76 years). In statistical tests a $P$-value of $<0.01$ was taken as evidence to reject the null hypothesis; values above this were considered non-significant.

\section{Results}

Age at recruitment ranged from 37 to 72 in men with a median of 56 years, and in women age ranged from 35 to 76 with a median of 52 years. Ninety-nine per cent of participants were white and $20 \%$ had a university degree or equivalent. Mean BMI was $26.1 \mathrm{~kg} \mathrm{~m}^{-2}$ in men and $24.8 \mathrm{~kg} \mathrm{~m}^{-2}$ in women.

Table 1 shows mean measured and self-reported waist and hip measurements and WHR, and the mean differences between measured and self-reported values. Values for all of the differences between measured and self-reported circumferences ranged from negative to positive: that is, some participants overreported the value and others underreported the value for each variable.

On average, men underestimated their waist circumference by $3.1 \mathrm{~cm}$ and underestimated their hip measurement by $1.8 \mathrm{~cm}$. WHR was underestimated by $1.12 \%$. Women, on average, underestimated their waist circumference by $1.9 \mathrm{~cm}$ and their hip measurement by $1.2 \mathrm{~cm}$. They underestimated their WHR by $0.94 \%$. There was evidence of a difference between the mean measured and selfreported values $(P<0.01)$ in each case, for men and for women.

Spearman's rank correlation coefficients for selfreported and measured waist measurements were 0.80 for men and 0.83 for women. The correlation coefficients for hip measurements were 0.74 for men and 0.86 for women. The correlation coefficients for WHR were 0.44 for men and 0.62 for women.

Table 2 shows the mean differences between measured and self-reported values according to selected anthropometric measurements and age among men. There was evidence ( $P<0.01$ for heterogeneity) that the accuracy of waist circumference varied with measured waist circumference and with BMI. There was on average greater underreporting of waist for those with a larger waist measurement and higher BMI. On average, those in the lowest tertile of waist measurement overestimated their waist circumference. The accuracy of hip self-reporting varied with measured hip circumference and with age: the greater the hip measurement or higher the BMI, the greater the mean value of underreporting. The accuracy of the WHR varied with measured WHR, age and BMI such that, on average, discrepancy between self-reported and measured ratio was greater for those with a larger ratio, for older participants and for those with greater BMI. Those in the lowest third of WHR or BMI overestimated their WHR on average.

Among women (Table 3), there was evidence $(P<0.01$ for heterogeneity) that the accuracy of waist circumference varied according to measured waist, age and BMI: those with a larger waist measurement underreported waist to a greater extent and older or heavier women also underreported to a greater extent on average. The

Table 1 Mean (SD) measured and self-reported anthropometric measurements and their differences

\begin{tabular}{lccccc}
\hline Variable & Measured & Self-reported & Difference $^{\star}$ & Percentage difference† & $t$-test \\
\hline Men $(n=1588)$ & & & & & \\
Waist $(\mathrm{cm})$ & $94.6(9.6)$ & $91.6(8.2)$ & $3.1(5.6)$ & $3.0(5.8)$ & $P<0.01$ \\
Hip $(\mathrm{cm})$ & $103.2(6.3)$ & $101.4(7.4)$ & $1.8(4.9)$ & $1.7(4.7)$ & $P<0.01$ \\
WHR & $0.92(0.06)$ & $0.90(0.06)$ & $0.01(0.06)$ & $1.1(6.7)$ & $P<0.01$ \\
Women $(n=2904)$ & & & & \\
Waist $(\mathrm{cm})$ & $79.5(10.6)$ & $77.6(10.4)$ & $1.9(5.4)$ & $2.2(6.4)$ & $P<0.01$ \\
Hip $(\mathrm{cm})$ & $102.3(9.2)$ & $101.1(9.3)$ & $1.2(4.5)$ & $1.1(4.5)$ & $P<0.01$ \\
WHR & $0.78(0.07)$ & $0.77(0.06)$ & $0.01(0.06)$ & $0.9(7.2)$ & $P<0.01$
\end{tabular}

SD - standard deviation; WHR - waist-to-hip ratio.

* Difference calculated as (measured minus self-reported) values. A positive value indicates underreporting on average by the participants, a negative value indicates overreporting on average by the participants.

†Percentage difference calculated as the difference (measured minus self-reported) as a percentage of the measured value. 
Table 2 Mean differences between measured and self-reported waist, hip and WHR, by tertile of measured value, age group and measured BMI, among men

\begin{tabular}{|c|c|c|c|c|c|c|c|c|}
\hline Waist & $n$ & Difference $^{*}(\mathrm{SD})$ & Нip & $n$ & Difference* $^{*}(S D)$ & WHR & $n$ & $\begin{array}{c}\text { Percentage } \\
\text { difference† (SD) }\end{array}$ \\
\hline Measured waist $(\mathrm{cm})$ & & & Measured hip $(\mathrm{cm})$ & & & Measured WHR & & \\
\hline $70-<91$ & 561 & $-0.29(4.18)$ & $86-<101$ & 548 & $0.80(4.46)$ & $0.73-<0.89$ & 532 & $-3.04(0.05)$ \\
\hline $91-<99$ & 526 & $3.54(4.19)$ & $101-<106$ & 533 & $1.98(4.80)$ & $0.89-<0.94$ & 527 & $1.63(0.05)$ \\
\hline $99+$ & 501 & $6.36(6.28)$ & $106+$ & 507 & $2.63(5.25)$ & $0.94+$ & 529 & $4.81(0.06)$ \\
\hline Test for heterogeneity & & $P<0.01$ & & & $P<0.01$ & & & $P<0.01$ \\
\hline Age group (years) & & & Age group (years) & & & Age group (years) & & \\
\hline $35-49$ & 460 & $3.39(6.12)$ & $35-49$ & 460 & $3.12(5.59)$ & $35-49$ & 460 & $0.03(0.07)$ \\
\hline $50-59$ & 541 & $3.10(5.33)$ & $50-59$ & 541 & $1.75(4.52)$ & $50-59$ & 541 & $1.23(0.06)$ \\
\hline $60-76$ & 587 & $2.82(5.54)$ & $60-76$ & 587 & $0.76(4.37)$ & $60-76$ & 587 & $1.89(0.06)$ \\
\hline Test for heterogeneity & & NS & & & $P<0.01$ & & & $P<0.01$ \\
\hline BMI $\left(\mathrm{kg} \mathrm{m}^{-2}\right)$ & & & BMI $\left(\mathrm{kg} \mathrm{m}^{-2}\right)$ & & & BMI $\left(\mathrm{kg} \mathrm{m}^{-2}\right)$ & & \\
\hline $17.8-<24.9$ & 528 & $0.79(4.56)$ & $17.8-<24.9$ & 528 & $1.63(4.63)$ & $17.8-<24.9$ & 528 & $-1.11(0.06)$ \\
\hline $24.9-<27.5$ & 527 & $3.00(4.99)$ & $24.9-<27.5$ & 527 & $1.61(4.49)$ & $24.9-<27.5$ & 527 & $1.40(0.06)$ \\
\hline $27.5+$ & 527 & $5.43(6.27)$ & $27.5+$ & 527 & $2.11(5.49)$ & $27.5+$ & 527 & $3.06(0.06)$ \\
\hline Test for heterogeneity & & $P<0.01$ & & & NS & & & $P<0.01$ \\
\hline
\end{tabular}

WHR - waist-to-hip ratio; BMI - body mass index; SD - standard deviation; NS - not significant.

* Difference calculated as (measured minus self-reported) values. A positive value indicates underreporting on average by the participants, a negative value indicates overreporting on average by the participants.

$\dagger$ Percentage difference calculated as the difference (measured minus self-reported) as a percentage of the measured value.

accuracy of hip circumference varied with measured hip and with age, such that younger women and those with a larger hip measurement underreported to a greater extent. There was no association of the extent of underreporting of hip circumference according to BMI. Accuracy of the WHR varied with measured WHR, age and BMI, such that women with higher WHR, older women or heavier women underreported to a greater extent. Those in the lowest third of WHR or the lowest age group overreported their WHR on average.

Table 4 shows the percentages of participants whose self-reported measurements assigned them to the correct tertile of anthropometric variable as calculated from the measured values. This was over $65 \%$ for waist and hip measurements among men and women. However, the percentage of accurate classification was lower for WHR, at $50.0 \%$ in men and $59.6 \%$ in women. Table 5 shows the percentages of participants whose self-reported measurements assigned them to the lowest tertile if the measured value assigned them to the highest tertile, and vice versa. This was low for waist and hip measurements: among men, only $1.5 \%$ were incorrectly categorised in this way according to waist measurement and $3.7 \%$ of self-reported hip measurements resulted in this misclassification. Among women this misclassification was less than $2 \%$ for waist and hip. However, for WHR, 10.8\% of men's

Table 3 Mean differences between measured and self-reported waist, hip and WHR, by tertile of measured value, age group and measured BMI, among women

\begin{tabular}{|c|c|c|c|c|c|c|c|c|}
\hline Waist & $n$ & Difference* $(S D)^{*}$ & Hip & $n$ & Difference* $(S D)$ & WHR & $\mathrm{n}$ & $\begin{array}{c}\text { Percentage } \\
\text { difference† (SD) }\end{array}$ \\
\hline Measured waist (cm) & & & Measured hip (cm) & & & WHR & & \\
\hline $59-<75$ & 1049 & $0.16(3.70)$ & $67-<99$ & 1036 & $0.24(4.24)$ & $0.59-<0.75$ & 977 & $-1.90(0.04)$ \\
\hline $75-<83$ & 951 & $2.18(4.98)$ & $99-<105$ & 907 & $1.48(4.27)$ & $0.75-<0.79$ & 960 & $0.54(0.05)$ \\
\hline $\begin{array}{l}83+ \\
\text { Test for heterogeneity }\end{array}$ & 904 & $\begin{array}{c}3.70(6.80) \\
P<0.01\end{array}$ & $105+$ & 961 & $\begin{array}{c}2.04(4.67) \\
P<0.01\end{array}$ & $0.79+$ & 967 & $\begin{array}{l}4.22(0.08) \\
P<0.01\end{array}$ \\
\hline Age group (years) & & & Age group (years) & & & Age group (years) & & \\
\hline $35-49$ & 1121 & $1.30(5.07)$ & $35-49$ & 1121 & $1.61(4.56)$ & $35-49$ & 1121 & $-0.16(0.06)$ \\
\hline $50-59$ & 1050 & $2.33(5.55)$ & $50-59$ & 1050 & $1.32(4.31)$ & $50-59$ & 1050 & $1.34(0.06)$ \\
\hline $60-76$ & 733 & $2.29(5.73)$ & $60-76$ & 733 & $0.49(4.43)$ & $60-76$ & 733 & $2.06(0.06)$ \\
\hline Test for heterogeneity & & $P<0.01$ & & & $P<0.01$ & & & $P<0.01$ \\
\hline BMI $\left(\mathrm{kg} \mathrm{m}^{-2}\right)$ & & & BMI $\left(\mathrm{kg} \mathrm{m}^{-2}\right)$ & & & BMI $\left(\mathrm{kg} \mathrm{m}^{-2}\right)$ & & \\
\hline $15.6-<23.4$ & 966 & $1.12(4.26)$ & $15.6-<23.4$ & 966 & $1.18(3.84)$ & $15.6-<23.4$ & 966 & $0.13(0.07)$ \\
\hline $23.4-<26.5$ & 964 & $1.65(5.10)$ & $23.4-<26.5$ & 964 & $1.38(4.44)$ & $23.4-<26.5$ & 964 & $0.56(0.06)$ \\
\hline $26.5+$ & 964 & $2.97(6.54)$ & $26.5+$ & 964 & $1.13(5.03)$ & $26.5+$ & 964 & $2.11(0.07)$ \\
\hline Test for heterogeneity & & $P<0.01$ & & & NS & & & $P<0.01$ \\
\hline
\end{tabular}

WHR - waist-to-hip ratio; BMI - body mass index; SD - standard deviation; NS - not significant.

* Difference calculated as (measured minus self-reported) values. A positive value indicates underreporting on average by the participants, a negative value indicates overreporting on average by the participants.

†Percentage difference calculated as the difference (measured minus self-reported) as a percentage of the measured value. 
Table 4 Percentage of participants whose self-reported measurement assigned them to the correct tertile

\begin{tabular}{lcc}
\hline Variable & Men & Women \\
\hline Waist & 66.9 & 69.8 \\
Hip & 65.9 & 73.4 \\
WHR & 50.0 & 59.6 \\
\hline
\end{tabular}

WHR - waist-to-hip ratio.

Table 5 Percentage of participants whose self-reported measurement incorrectly assigned them to the lowest tertile if in the highest tertile, or the highest tertile if in the lowest tertile

\begin{tabular}{lrc}
\hline Variable & Men & Women \\
\hline Waist & 1.5 & 1.2 \\
Hip & 3.7 & 1.4 \\
WHR & 10.8 & 6.8
\end{tabular}

WHR - waist-to-hip ratio.

measurements and $6.8 \%$ of women's measurements resulted in this misclassification.

\section{Discussion}

In this study of 4492 EPIC-Oxford participants there were high correlations and small discrepancies on average between self-reported and measured values for waist and hip circumferences. The small magnitude of the underreporting gives confidence that self-reported waist and hip measurements are sufficiently accurate to be of use in epidemiological studies examining the influence of anthropometric measures on disease outcomes. However, systematic biases were identified such that, overall, the magnitude of underreporting increased with body size and, in some cases, with age.

Previously, self-reported and measured height and weight data in EPIC-Oxford were compared ${ }^{10}$ : those with higher body weight tended to underestimate their weight and BMI to a greater extent than those with lower body weight, and shorter individuals tended to overestimate their height to a greater extent. The data reported here further support the idea of a bias in reporting on body size and shape corresponding to a perceived healthy or fashionable standard, as has been noted previously ${ }^{11}$. These biases are likely to attenuate risk estimates of the relationship between body size and shape and disease, but the results presented here suggest that the degree of attenuation would be small.

Assuming that similar biases existed in the overall cohort, it would be possible to use the data presented here to adjust values for waist and hip measurements for other EPIC-Oxford participants for whom measured values are not available, taking account of the factors associated with the discrepancies. This has been shown for correcting selfreported height and weight in the same cohort ${ }^{10}$. This may be done to adjust values after completing an analysis so as to determine accurate information to be used in public health recommendations.
For the variable WHR, derived from waist and hip measurements, the correlation was lower than for waist or hip measurements and the percentage of incorrect classification was somewhat higher, although the average discrepancy in this variable was less than $2 \%$. This study suggests that errors in the individual waist and hip measurements are compounded in this variable, indicating that using WHR derived from self-reported measurements may be more likely to obscure true associations than using either waist or hip measurement alone and outcome. The physiological meaning of differences in WHR is difficult to interpret and the individual waist and hip circumferences may be of more use in defining health risk.

To our knowledge, only seven other studies have published results on the accuracy of self-reported waist and hip measurements ${ }^{2-8}$ and the study presented here is by far the largest. The majority of previous studies (all but one performed among participants in the USA) have shown that, on average, waist and hip circumferences were underestimated using self-reported data. One study of US men found that waist was overestimated by 0.41 $\mathrm{cm}^{7}$. The greatest underestimation was $6.4 \mathrm{~cm}$ by men in a Scottish study ${ }^{8}$. For hip circumference the discrepancy ranged from an underestimation of $1.98 \mathrm{~cm}$ in a study of US men ${ }^{4}$ to an underestimation of $6.1 \mathrm{~cm}$ in the study of Scottish men ${ }^{8}$. The average discrepancies found here were within the range of previous reports.

Differences in study protocols may account for the slight variation in study findings. In some previous studies instructions and a tape measure were sent to each participant ${ }^{3,6,7}$. In the EPIC-Oxford questionnaire, no instructions as to how to make the waist and hip measurements were given. It is possible that participants recorded circumferences according to their clothing size, or recorded measures made some time ago. In one previous study the self-reported measurement was made in the same interview during which the measured values were taken ${ }^{2}$, whereas in the study presented here, the measurement by the nurse was within a few weeks (usually within three weeks). It is possible that agreement would have been even higher had instructions as to how to take the measures been given, or the time interval between measures been shorter; however, despite these limitations, acceptable results were obtained.

In this study, men underreported waist to a greater extent than did women and the percentage misclassification of hip measurement was higher among men than among women. This may be due to the distribution of BMI being higher among men than among women in this study group. Alternatively, it may indicate that the women in this study were more familiar with making these measurements.

Waist and hip circumferences were assessed in EPICOxford with the aim of examining associations between these exposures and subsequent risk of disease and mortality. This study suggests that the self-reported data are sufficiently accurate for this purpose. 


\section{Acknowledgements}

We thank all participants in EPIC-Oxford and all scientists and clerical staff who have worked on these studies. EPIC is supported by the Europe Against Cancer Programme of the Commission of the European Communities. This analysis was supported by Cancer Research UK and the Medical Research Council.

\section{References}

1 Han TS, van Leer EM, Seidell JC, Lean ME. Waist circumference action levels in the identification of cardiovascular risk factors: prevalence study in a random sample. British Medical Journal 1995; 311(7017): 1401-5.

2 Hall TR, Young TB. A validation study of body fat distribution as determined by self-measurement of waist and hip circumference. International Journal of Obesity 1989; 13(6): 801-7.

3 Kushi LH, Kaye SA, Folsom AR, Soler JT, Prineas RJ. Accuracy and reliability of self-measurement of body girths. American Journal of Epidemiology 1988; 128(4): 740-8.

4 Rimm EB, Stampfer MJ, Colditz GA, Chute CG, Litin LB, Willett WC. Validity of self-reported waist and hip circumferences in men and women. Epidemiology 1990; 1(6): 466-73

5 Weaver TW, Kushi LH, McGovern PG, Potter JD, Rich SS,
King RA, et al. Validation study of self-reported measures of fat distribution. International Journal of Obesity and Related Metabolic Disorders 1996; 20(7): 644-50.

6 Freudenheim JL, Darrow SL. Accuracy of self-measurement of body fat distribution by waist, hip, and thigh circumferences. Nutrition and Cancer 1991; 15(3-4): 179-86.

7 Roberts CA, Wilder LB, Jackson RT, Moy TF, Becker DM. Accuracy of self-measurement of waist and hip circumference in men and women. Journal of the American Dietetic Association 1997; 97(5): 534-6.

8 Han TS, Lean ME. Self-reported waist circumference compared with the 'Waist Watcher' tape-measure to identify individuals at increased health risk through intra-abdominal fat accumulation. British Journal of Nutrition 1998; 80(1): $81-8$.

9 Davey GD, Spencer EA, Appleby PN, Allen NE, Knox KH, Key TJ. EPIC-Oxford: lifestyle characteristics and nutrient intakes in a cohort of 33883 meat-eaters and 31546 non meat-eaters in the UK. Public Health Nutrition 2003; 6(3): 259-68.

10 Spencer EA, Appleby PN, Davey GK, Key TJ. Validity of selfreported height and weight in 4808 EPIC-Oxford participants. Public Health Nutrition 2002; 5(4): 561-5.

11 Ziebland S, Thorogood M, Fuller A, Muir J. Desire for the body normal: body image and discrepancies between self reported and measured height and weight in a British population. Journal of Epidemiology and Community Health 1996; 50(1): 105-6. 